\title{
Fomento de hábitos saludables a través de la coordinación del profesorado de educación física
}

\section{Promoting healthy habits through the coordination of physical education teachers}

\author{
I. García-Martínez ${ }^{1}$, P. Puertas-Molero², G. González-Valero ${ }^{2 *}$, M. Castro-Sánchez ${ }^{3}$ e I. Ramírez-Granizo² \\ 1 Facultad de Ciencias de la educación. Dpto. Didáctica y Organización Escolar. Universidad de Granada \\ 2 Facultad de Ciencias de la Educación. Dpto. Didáctica de la expresión Musical, Plástica y Corporal. Universidad de Granada \\ 3 Universidad Internacional de La Rioja.
}

\begin{abstract}
Resumen: La presente investigación tiene por objetivo analizar el grado de coordinación pedagógica existente entre el profesorado de educación física, a partir de la promoción de un estilo de vida saludable entre el alumnado. En concreto, se trató de determinar si existía o no una tendencia a trabajar colaborativamente en aras a conseguir unos objetivos específicos en el área de Educación Física. Para ello, se realizó un cuestionario elaborado para esta investigación, que estaba compuesto por 25 ítems, dirigido a maestros con la especialidad de educación física en ejercicio. A partir de un muestreo aleatorio simple, se consiguieron 300 respuestas de maestros de la provincia de Granada. Entre los resultados, destaca la importancia de la motivación en la conjugación del desempeńo profesional docente del especialista del área de educación física. De este modo, se aprecia una fuerte correlación existente entre motivación e inclusión social y vida saludable, así como entre motivacióne innovación curricular.

Palabras clave: Coordinación docente; Profesorado de educación física; educación saludable; educación primaria
\end{abstract}

Abstract: The aim of this research is to analyze the degree of pedagogical coordination existing among physical education teachers, based on the promotion of a healthy lifestyle among the students. Specifically, an attempt was made to determine whether or not there was a tendency to work collaboratively in order to achieve specific objectives in the area of Physical Education. To do this, a questionnaire was prepared for this research, which was composed of 25 items, aimed at teachers with the specialty of physical education in practice. From a simple random sampling, 300 responses were obtained from teachers in the province of Granada. Among the results, it emphasizes the importance of motivation in the conjugation of the professional teaching performance of the specialist in the area of physical education. In this way, a strong correlation exists between motivation and social inclusion and healthy life, as well as between motivation and curricular innovation.

Keywords: Teacher Collaboration; Physical education teachers; Health education; Secondary Education.

\section{Introducción}

Lograr la mejora escolar continúa siendo una de las prioridades de las escuelas. Estudios anteriores han apuntado hacia la profesionalización del profesorado (Martín-Romera y García-Martínez, 2018; Hargreaves y Fullan, 2014; Montecinos, Pine, Campos-Martínez, Domínguez y Carreño, 2014; Seashore-Louis, 2007) como vía a seguir para conseguirlo. Poner el foco en el quehacer docente para conseguir un efecto positivo en el aprendizaje del alumnado, pasa por diversos factores. Entre los factores externos al profesorado pero que tienen una incidencia notable sobre su desempeño profesional (Murillo, 2008; Sánchez, González, López, \& Díaz, 2018), destacan los factores organizacionales (Wood, 2011), que atienden al funcionamiento del centro, al establecimiento de un proyecto común, la disposición de unas condiciones idóneas para esbozar prácticas de colaboración entre el personal (...). En cuanto a los intrapersonales (Ful-

Dirección para correspondencia [Correspodence address]: G. GonzálezValero. Facultad de Ciencias de la Educación. Dpto. Didáctica de la expresión Musical, Plástica y Corporal. Universidad de Granada (España). E-mail: ggvalero@ugr.es lan, 2010), la atribución de la etiqueta de buen docente va a depender de factores como la motivación y la vocación de estos profesionales, así como de su compromiso y disposición (positiva) hacia la mejora de sus prácticas docentes y la colaboración (Fernández-Argüelles y González-González de Mesa, 2018). Finalmente, existen otros factores que determinan la calidad de estos procesos en el aula (Elmore, 2010) y que han sido recogidos en la presente investigación, como son la innovación del currículum, la motivación o la disciplina.

Independientemente de eso, autores como Gibbins y Cob (2017), señalan una serie de efectos, que son consecuenciadel establecimiento de aprendizajes profesionales en el seno de la organización escolar:

- El aprendizaje profesional demanda que las oportunidades de aprendizaje sean intensas y prolongadas en el tiempo. Igualmente, para que este aprendizaje de sus frutos sobre los procesos de aprendizaje del alumnado, es necesario que el docente innove en sus prácticas.

- El ejercicio docente y los intentos de mejora deben fundamentarse en los problemas a los que se enfrenta el profesorado en su quehacer diario. 
- Los docentes deben enfocar su enseñanza al pensamiento del estudiante, diseńando sus planteamientos metodológicos según las dificultades que éstos vayan teniendo. Se trata de detenerse en el proceso, sin obcecarse en el resultado, para ajustar la instrucción gradualmente a sus necesidades.

- El aprendizaje profesional trae consigo el desarrollo de las comunidades de docentes, que trabajan conjuntamente en pos a un bien común (el aprendizaje del alumnado), sorteando las dificultades que van encontrando a partir del diálogo, el apoyo y el respeto mutuos.

- Elaprendizaje profesional docente está ligado al denominado "aprendizaje activo", que supone innovar a través de la reflexión que emana de las prácticas de aula. Aspecto que precisamente le ayuda a mejorar sus prácticas y crecer profesionalmente.

En esta línea también cabalga el estudio de Cravensy Drake (2017), quiénes a partir de un proyecto de colaboración China-EE.UU., diseñaron un modelo para implementar y escalar los Grupos de excelencia entre pares del maestro (TPEG), en el que se agrupaba al profesorado según la materia o el curso (coordinación vertical y horizontal, respectivamente). El profesorado participante debía estar realmente implicado en la creación de comunidades de prácticas para la mejora escolar. En palabras de estos autores, el TPEG es un modelo que "Facilita la profesionalización de la enseñanza a través de la desprivatización de la práctica docente, la planificación colaborativa, el dar y recibir retroalimentación procesable, y responsabilizarse reciprocamente por la implementación de medidas de mejora" (p. 348).

Bajo estos supuestos, es decir, la colaboración docente para la mejora escolar, la necesaria coordinación en la organización escolar y el deseo y motivación de mejora por parte del profesorado, se inscribe la investigación que a continuación se detalla.

\section{Metodología}

\section{Diseño, procedimiento y muestra}

Para el presente trabajo estudio se realizó un estudio descriptivo de corte transversal con una muestra representativa de maestros de la especialidad de educación física en educación primaria. A partir de la literatura existente sobre la temática objeto de estudio, se confeccionó un cuestionario online ad hoc de 25 ítems, de 4 opciones de respuesta, con la herramienta de Google Form.

Para obtener los datos, se envió un email al correo electrónico institucional de los colegios solicitando la colaboración al presente estudio, donde se les explicaba en qué consistía. En paralelo, también se colgó el enlace del cuestionario en diversos foros de profesorado. Tras este proceso, la muestra constituyente del estudió se configuró con un total de 300 profesores, siendo el $64.33 \%$ hombres y el $35.66 \%$ mujeres. Todos los docentes participaron de forma voluntaria, respetando siempre el acuerdo de ética de investigación de Helsinki, además de ser informados del anonimato de las respuestas y el carácter científico del estudio.

\section{Análisis estadístico}

El análisis de los datos se realizó mediante el programa estadístico SPSS ${ }^{\circ}$ 24.0. Los descriptivos básicos se presentan mediante frecuencias, mostrando la media y desviación típica hallada en los ítems seleccionados. Asimismo, también se muestran los resultados del análisis diferencial realizados, atendiendo al sexo y a la experiencia de los individuos. También se exponen las tablas de contingencia para expresar los análisis relacionales realizados. Para conocer la consistencia interna de cada dimensión se utilizó el Alfa de Cronbach, cuya puntuación fue de 0.895 . La asociación entre variables se determinó a través del test Chi-cuadrado de Pearson, estableciéndose la significación en 0,05.

\section{Resultados}

Los resultados conseguidos tras la elaboración de los análisis descriptivos, que se muestran en la tabla 1 , señalan que de los 300 profesores los varones eran mayoría (57.3\%) frente a las mujeres (42.7\%). En paralelo, también se hizo una distinción entre enseńanza pública y privada-concertada, encontrando que el $76.66 \%$ procedía de loscentros de enseñanza pública y el restante $23.33 \%$ de centrosprivados-concertados.

Tabla 1. Descriptivos de las variables.

\begin{tabular}{lcc}
\hline Género & & \\
\hline Masculino & $57.3 \%$ & $(\mathrm{n}=172)$ \\
Femenino & $42.7 \%$ & $(\mathrm{n}=128)$ \\
\hline Centro de Procedencia & & \\
\hline Público & $76.66 \%$ & $(\mathrm{n}=230)$ \\
Concertado-Privado & $23.33 \%$ & $(\mathrm{n}=70)$ \\
\hline
\end{tabular}

Como se detalla en la tabla 1, en los individuos participantes en el estudio se visibiliza una superioridad de los hombres respecto a las mujeres, por lo que puede decirse que aún existe cierta tendencia en favor de los hombres, en desempeñar la función de profesor especialista de educación física.

Además del sexo y el centro de procedencia, se tuvo presente los ańos de ejercicio docente del profesorado, con la finalidad de contextualizar sus respuestas y conocer en qué medida éstas son reflejo de su experiencia. 
Para unificar este factor, se optó por agruparlo en "menos de 5 años", "entre 5 y 15 años" y "más de 5 años", tal y como se expone a continuación:

Tabla 2. Muestra según años de experiencia.

\begin{tabular}{lcc}
\hline Experiencia docente & $\mathbf{n}$ & $\%$ \\
\hline Menos de 5 años & 121 & 40.3 \\
Entre 5 y 15 años & 121 & 37.3 \\
Más de 15 ańos & 67 & 22.3 \\
\hline
\end{tabular}

Existe una clara superioridad en la tasa de respuesta del profesorado cuya experiencia se remonta a "menos de 5 años" y "entre 5 y 15 ańos", con 121 participantes cada uno, frente al profesorado más experimentado, cuya representación decrece a solo 67 participantes.

Una vez delimitadas las características sociodemográficas más notorias, se procede a exponer los resultados descriptivos y diferenciales del cuestionario.

El análisis de frecuencias realizado, muestra que las puntuaciones del profesorado en estas cuestiones se sitúan entre "bastante" y "mucho". A su vez, también se observa una gran variabilidad en las respuestas, que va desde 0.475 a 0.793 . De todos ellos, la cuestión más valorada por los participantes es "Usted habla con sus compañeros sobre cuestiones relacionadas con problemas de disciplina y control de conducta de los alumnos", con una puntuación media de 3.66. Esta puntuación refleja como las conductas disruptivas aún siguen siendo una preocupación constante en el profesorado para poder desempeñar su labor. De hecho, examinando la tabla se observa como este ítem es el que obtiene una desviación típica claramente inferior a la mayoría, por lo que se puede determinar que existe cierto consenso a la hora de señalar a la disciplina como una temática de preocupación entre el profesorado. En el lado opuesto se sitúa la cuestión "Usted habla con sus compañeros sobre cómo fomentar en los alumnos el desarrollo de valores ciudadanos y hábitos saludables", con una puntuación media de 3.07. Aunque sigue siendo una puntuación medianamente elevada, este aspecto resulta preocupante si lo que se pretende es iniciar y consolidar en el alumnado hábitos de vida saludables, que le permitan desarrollar conductas sostenibles.

Tabla 3. Descriptivos de las variables.

\begin{tabular}{|c|c|c|}
\hline Ítem & M & DT \\
\hline 1. Usted debate con sus compañeros acerca de la línea educativa a seguir & 3.17 & .664 \\
\hline 2. Usted habla con sus compañeros sobre cómo fomentar en los alumnos el desarrollo de valores ciudadanos y hábitos saludables. & 3.07 & .712 \\
\hline 3. Usted habla con sus compañeros sobre cómo despertar la motivación del alumnado hacia el deporte. & 3.17 & .765 \\
\hline 4. Usted habla con sus compañeros sobre cuestiones relacionadas con problemas de disciplina y control de conducta de los alumnos. & 3.66 & .475 \\
\hline 5. Usted habla con sus compañeros sobre cuestiones relacionadas con la innovación del currículum. & 3.40 & .793 \\
\hline 6. Usted debate con sus compañeros sobre las metodologías a emplear & 3.46 & .608 \\
\hline
\end{tabular}

De otro lado, las cuestiones estrictamente pedagógicas y de innovación, presentes en los ítems "Usted debate con sus compañeros sobre las metodologías a emplear ", y "Usted habla con sus compañeros sobre cuestiones relacionadas con la innovación del currículum", ascienden a valores medios de 3.46 y 3.40, respectivamente. Las puntuaciones elevadas sobre estos aspectos íntimamente ligados a la coordinación y colaboración docentes, invitan, aunque con cautela, a desarrollar una visión optimista sobre que existen indicios de colaboración y diálogo en los centros de educación primaria.

Además de conocer la tendencia de las respuestas de los encuestados, interesaba analizar si existían diferencias en relación con el sexo. Para ello, se realizó la prueba $\mathrm{T}$ para muestras independientes, arrojando los siguientes resultados:

Tabla 4. Resultados de las dimensiones según el sexo.

\begin{tabular}{|c|c|c|c|c|c|}
\hline & & M & DT & $\mathrm{F}$ & Sig. \\
\hline \multirow{2}{*}{ ISVS } & Hombres & 3,05 & 0,711 & \multirow{2}{*}{-0.498} & \multirow{2}{*}{.619} \\
\hline & Mujeres & 3,09 & 0,715 & & \\
\hline \multirow{2}{*}{ Mot } & Hombres & 3.12 & 0.751 & \multirow{2}{*}{-1.347} & \multirow{2}{*}{.0179} \\
\hline & Mujeres & 3.24 & 0.781 & & \\
\hline \multirow{2}{*}{$\mathrm{D}$} & Hombres & 3.66 & 0.474 & \multirow{2}{*}{0.118} & \multirow{2}{*}{.0906} \\
\hline & Mujeres & 3.66 & 0.477 & & \\
\hline \multirow{2}{*}{ IC } & Hombres & 3.31 & 0.571 & \multirow{2}{*}{$-1,293$} & \multirow{2}{*}{.0197} \\
\hline & Mujeres & 3.40 & 0.594 & & \\
\hline
\end{tabular}

ISVS: Inclusión Social y Vida Saludable; Mot: Motivación; D: Disciplina; IC: Innovación Curricular. 
Dado que la prueba de Levene fue menor a 0.05 en todos los casos, se asumió que las varianzas son iguales.

Por lo general no se observan diferencias significativas en ninguna de las dimensiones respecto al sexo, obteniendo significaciones entre 0.096 y 0.619. A grandes rasgos, las mujeres obtienen medias ligeramente superiores en todas las dimensiones, salvo en la dimensión Disciplina, donde se obtienen puntuaciones iguales $(M=3.66)$. La diferencia en la tasa de respuesta es superior para la dimensión Motivación, donde las mujeres otorgan una puntuación media de 3.24, frente a 3.12 de los hombres. En relación con la variabilidad de las respuestas, se observan índices de dispersión que oscilan entre 0.571 (hombres)/0.594(mujeres) en la dimensión Innovación Curricular y 0.751 (hombres)/0.781 (mujeres) en Motivación.

$\mathrm{Al}$ igual que con el sexo, también se indagó sobre el posible impacto que podría tener los años de desempeño profesional en la percepción del profesorado sobre la coordinación y colaboración docente, entre otras cuestiones. Los resultados hallados a partir de la ANOVA de un factor, se exponen a continuación.

Tabla 5. Resultados de las dimensiones según la experiencia.

\begin{tabular}{cccccc}
\hline & & M & DT & F & Sig. \\
\hline \multirow{3}{*}{ ISVS } & menos de 5 ańos & 3,05 & 0,72 & & \\
& Entre 5 y 15 & 3,08 & 0,71 & 0,086 &, 917 \\
& más de 15 & 3,09 & 0,69 & & \\
\hline \multirow{2}{*}{ Mot } & menos de 5 ańos & 3,16 & 0,79 & & \\
& Entre 5 y 15 & 3,21 & 0,77 & 0,274 &, 761 \\
& más de 15 & 3,13 & 0,69 & & \\
\hline \multirow{2}{*}{ D } & menos de 5 años & 3,68 & 0,46 & & \\
& Entre 5 y 15 & 3,68 & 0,46 & 0,759 &, 469 \\
& más de 15 & 3,60 & 0,49 & & \\
\multirow{2}{*}{ IC } & menos de 5 ańos & 3,33 & 0,59 & & \\
& Entre 5 y 15 & 3,34 & 0,58 & 0,196 &, 822 \\
& más de 15 & 3,38 & 0,55 & & \\
\hline
\end{tabular}

ISVS: Inclusión Social y Vida Saludable; Mot: Motivación; D: Disciplina; IC: Innovación Curricular.
Los resultados mostrados en la tabla anterior muestran que no se han encontrado diferencias estadísticamente significativas en las dimensiones analizadas respecto a la variable experiencia docente (valores de prueba entre 0.469 y 0.917 ). A la luz de los resultados, se aprecian puntuaciones similares en cada una de las dimensiones analizadas. Particularmente, en la dimensión Inclusión Social y Vida Saludable, los docentes más experimentados obtienen puntuaciones sensiblemente superiores $(M=3.09)$ al resto. Esta tendencia se repite con la dimensión Innovación Curricular, donde califican 3.38, frente a 3.33 (menos de 5 ańos) y 3.34 (entre 5 y 15 ańos). Por el contrario, en las dimensiones Motivación y Disciplina, el sector que obtiene puntuaciones más altas se ubica en docentes con experiencias entre 5 y 15 ańos, con medias de 3.21 y 3.68, respectivamente.

Ahondando en las relaciones entre los ítems analizados en este trabajo (véase tabla 6), destaca la fuerte vinculación entre la inclusión social y vida saludable con la motivación, así como la motivación con la innovación curricular. Esto da pie a situar a la búsqueda de la motivación como la base sobre la que los maestros encuestados vertebran sus prácticas docentes, con la intención de obtener una mayor implicación del alumnado, y, en consecuencia, resultados académicos superiores.

Tabla 6. Matriz de correlaciones entre elementos.

\begin{tabular}{lcccc}
\hline & Inclusión social y vida saludable & Motivación & Disciplina & Innovación curricular \\
\hline Inclusión social y vida saludable & - &, 774 &, 409 &, 560 \\
Motivación &, 774 & - &, 566 &, 641 \\
Disciplina &, 409 &, 566 & - &, 533 \\
Innovación curricular &, 560 &, 641 &, 533 & - \\
\hline
\end{tabular}




\section{Discusión}

En la literatura encontramos estudios de naturaleza similar al que ocupa la presente investigación. Ejemplo de ello es el trabajo realizado por Kazemiy Hubbard (2008), quienes sostuvieron que la innovación en la enseñanza requiere de una reflexión en la práctica del aula, señalando que la viabilidad de las metodologías empleadas, solo es factible (y deseable) cuando se hace un contraste entre lo que se venía haciendo y lo nuevo, hallando cierta mejoría en el aprendizaje del alumnado. Defendiendo la fuerte relación existente entre coordinación docente, innovación educativa y mejora escolar, se sitúan una amplia variedad de investigaciones llevadas a cabo a nivel internacional (Hargreaves y Shirley, 2012; Hopkins, 2010; Sebastiany Allensworth, 2012;). Otras investigaciones han puesto el foco sobre el aprendizaje cooperativo en el área de educación física, resaltando la versatilidad y transversalidad de esta materia para emprender innovaciones metodológicas y curriculares, así como los beneficios y facilidades que reportan al aprendizaje de los alumnos (Navarro-Paton, Rodríguez-Fernández y Eirin, 2016; VelázquezCallado, 2015). Este autor aboga que la implementación de metodologías como el aprendizaje cooperativo favorece la resolución de conflictos, fomentando conductas prosociales entre el alumnado, al tiempo que desarrollan habilidades como la creatividad, el autoconcepto y despiertan la motivación hacia el deporte y hábitos saludables (Navarro-Paton, Rodríguez-Fernández y Eirin, 2016; Sevil-Serrano, AbarcaSos, Clemente, Murillo-Pardo y García-González, 2016).

Otros estudios han tratado de identificar si la variable sexo, ejercía influencia sobre el estilo de enseńanza del profesorado, la motivación o el planteamiento de un estilo de vida saludable. A diferencia del presente estudio, sus resultados sí quearrojaron relaciones significativas entre estas variables y el sexo. Ejemplo de ello es el estudio planteado por González-Peiteado (2013), quién planteó si existían diferencias en los estilos de enseñanza del alumnado de magisterio en función al sexo, encontrando diferencias estadísticamente significativas. En palabras de la autora "las mujeres puntúan más alto que los hombres en los estilos reflexivo, cooperador, individualizador, innovador e indagador, mientras que los hombres puntúan más alto en el estilo académico" (p. 63). El estudio desarrollado por Martínez-Galindo, Alonso, Cervelló, y Moreno (2009), contextualizado en el área de Educación Física, también tuvo en cuenta el sexo de los docentes a la hora de analizar los perfiles motivacionales del alumnado. Sus resultados destacan la relación significativa existente entre la variable sexo y el grado de implicación docente por mantener la disciplina y fomentar un clima motivacional en el aula, a favor de los hombres (Padial-Ruz, Ubago-Jiménez, Espejo-Garcés, Puertas-Molero, Chacón-Cuberos y Moreno-Arrebola,2017).

Investigaciones como la desarrollada por Bilbao-Villegas y Monereo-Font (2011), coinciden en apuntar a los asuntos relacionados con la gestión de clase y la actitud del alumnado frente al aprendizaje (motivación) como los incidentes críticos más destacados. Sin embargo, no hallaron diferencias significativas entre los maestros noveles y los experimentados.

En relación con ello, aunque en el presente estudio no se han obtenido resultados estadísticamente significativos cuando se analizaron las dimensiones en función a la experiencia docente, en la Innovación curricular, se obtuvo que a medida que incrementaban los años de experiencia, incrementaban las puntuaciones medias en innovación. En menos de 5 ańos, tenemos un 3.33; entre 5 y 15 años, una puntuación media de 3.34 y en más de 15 años de experiencia docente, se ha obtenido una media de 3.38. En la dimensión 3, Disciplina, se aprecian mayores puntuaciones de disciplina cuando la experiencia es menor. Respecto a la motivación, se hallan las puntuaciones más altas para los encuestados que tienen una experiencia de entre 5 y 15 ańos, seguidos de los docentes con una experiencia inferior a 5. En este caso, se aprecia como la motivación es inferior en los docentes con más de 15 años de experiencia, hecho que puede explicarse por el desgaste que suele provocar el ejercicio profesional y el síndrome de Burnout, a lo largo de los ańos

Por otro lado, un trabajo reciente de Krichesky y Murillo (2018) ha señalado, desde una perspectiva cualitativa, aquellas prácticas de colaboración más extendidas entre los docentes. En los resultados de su investigación, se halló que la colaboración docente era más visible en cuestiones que atañían a la coordinación, al desarrolloconjunto y a la resolución de problemas. Aunque esta investigación no se ubica en el contexto del profesorado especialista en el área de educación física, puede confirmarse la relación existente entre coordinación pedagógica (y docente) y mejora escolar, lo cual, se refleja en el rendimiento, la convivencia y la disciplina y la innovación curricular. Además, paradójicamente se puso de relieve que las prácticas de coordinación no eran sinónimo de aprendizaje docente, en contraposición al emprendimiento de proyectos interdisciplinares. De hecho, estos autores afirman que "el desarrollo de proyectos interdisciplinarios y la resolución conjunta de problemas, en cambio, demandan una fuerte interdependencia sobre la base de valores compartidos y se asientan en intercambios con una gran potencialidad para generar nuevos aprendizajes" (p. 136).

Estos hallazgos revelan que cuando se trata de interdisciplinariedad, se observan atisbos de colaboración entre el profesorado, lo que fortalece la idoneidad de hacer este tipo de estudio en profesorado especialista en educación física.

\section{Conclusiones}

Desarrollar prácticas educativas, fruto de una coordinación eficaz, es un garante de calidad en los procesos de enseñanza 
y aprendizaje. Si se busca construir aprendizajes significativos en el alumnado, es menester que el profesorado tome responsabilidad de su papel en esta ecuación, abriendo camino hacia el diseńo de metodologías y planteamientos innovadores, que hagan una distinción notaria respecto a la enseńanza tradicional. En este entramado, tal y como se ha evidenciado en este estudio, la motivación ocupa un lugar preponderante.

La transversalidad que acompaña al área de educación física obliga, en cierto modo, a ir más allá de la mera transmisión de conocimientos, dejando un espacio considerable a otros aspectos, como lo son la transmisión de unos valores afines a la obtención de unos hábitos saludables (Sevil-Serrano et al., 2016) o el aprendizaje de ciertos códigos relativos a la disci- plina, que faciliten la inclusión del alumnado en la sociedad.

Como se ha reflejado en los resultados obtenidos, el diseño y puesta en práctica de trabajos coordinados entre el profesorado de educación, así como con otros profesores de otras áreas, contribuye positivamente a la consecución de todos estos aspectos. No es cuestión de ir sumando cada una de las partes que componen el personal docente, más bien de lo que se trata es de ir trabajando colaborativamente, a partir del diálogo, del intercambio de experiencias y del consenso, ciertas pautas que den lugar a un aprendizaje profesional compartido en la institución en primera instancia y, unas condiciones óptimas que favorezcan aprendizajes de calidad entre el alumnado, en segundo término.

\section{Referencias}

1. Bilbao-Villegas, G., y Monereo-Font, C. (2011). Identificación de incidentes críticos en maestros en ejercicio: propuestas para la formación permanente. Revista electrónica de investigación educativa, 13(1), 135151

2. Cravens, X. y Drake, T. (2017). From Shanghai to Tennessee: Developing instructional leadership through Teacher Peer Excellence Groups. International Journal for Lesson and Learning Studies, 6(4), 348-364. https://doi.org/10.1108/IJLLS-12-2016-0062

3. Elmore, R.F. (2010). Mejorando la escuela desde la sala de clases. Santiago de Chile: Fundación Chile.

4. Fernández-Argüelles, D. y González-González de Mesa, C. (2018) Educación Física y AprendizajeCooperativo: una experiencia práctica. Journal of Sport and Health Research, 10(1), 43-64.

5. Fullan, M. (2010). All Systems Go: The Change Imperative for Whole System Reform. Thousands Oaks, CA: Sage.

6. Gibbins, L. K., y Cobb, P. (2017). Focusing on Teacher Learning Opportunities to Identify Potentially Productive Coaching Activities. JournalofTeacherEducation, 68(4) 411-425.

7. González-Peiteado, M. (2013). Los estilos de enseñanza y aprendizaje como soporte de la actividad docente. JournalofLearningStyles, 6(11), 5170 .

8. Hargreaves, A. y Fullan, M. (2014). Capital profesional: transformar la enseñanza en cada escuela. Madrid: Morata.

9. Hargreaves, A. y Shirley, D. (2012). The Global Fourth Way. The Quest for Educational Excellent. Thousand Oaks, CA: Corwin Press.

10. Kazemi, E., y Hubbard, A. (2008). New directions for the design and study of professional development: Attending to the coevolution of teachers' participation across contexts. Journal of Teacher Education, 59(5), 428-441

11. Hopkins, D. (2010). A Teacher's guide to Classroom Research. Maidenhead: Open University Press.

12. Krichesky, G. J. y Murillo F. J. (2018). La colaboración docente como factor de aprendizaje y promotor de mejora. Un estudio de casos. Educación XX1, 21(1), 135-156.Doi: 10.5944/educXX1.15080

13. Martín-Romera, A. y García-Martínez, I. (2018). Profesionalización del docente en la actualidad: Contribuciones al desarrollo profesional. Profesorado: Revista de curriculum y formación del profesorado, 22(1), 7-23.

14. Martínez-Galindo, C., Alonso, N., Cervelló, E., y Moreno, J. A. (2009). Perfiles motivacionales y disciplina en clases de educación física. Diferencias según las razones del alumnado para ser disciplinado y la per- cepción del trato generado por el profesorado en el aula. Cultura y Educación, 21(3), 331-343.

15. Murillo, F.J. (2008). Hacia un modelo de eficacia escolar. Estudios multinivel sobre los factores de eficacia en las escuelas españolas. Revista electrónica iberoamericana sobre Calidad, Eficacia y Cambio en Educación, 6(1), 4-28.

16. Montecinos, C., Pine, M., Campos-Martínez, J., Domínguez, R. y Carreño, C. (2014). Master teachers as professional developers: Managing conflicting versions of professionalism. Educational Management Administration \& Leadership, 42(2) 275-292.

17. Navarro-Paton, R., Rodríguez-Fernández, J. E. yEirin, R. (2016). Análisis de la satisfacción de las necesidades psicológicas básicas, motivación y disfrute en Educación Física en Primaria.Sportis, 3 (2), 439-455. DOI: http://dx.doi.org/10.17979/sportis.2016.2.3.1758.

18. Padial-Ruz, R.,Ubago-Jiménez, J.L., Espejo-Garcés, T., Puertas-Molero, P., Chacón-Cuberos, R. y Moreno-Arrebola, R. (2017). Promoción de hábitos saludables en elsegundo ciclo de educación infantil: una propuesta a través del movimiento.Trances, 9(4), 693-712.

19. Sánchez García, C., González Carcelén, C. M., López Sánchez, G. F., \& Díaz Suárez, A. (2017). Satisfaction of external customers. A case study of an indoor swimming pool. SPORT TK: Revista EuroAmericana de Ciencias del Deporte, 6(2), 81-88.

20. Seashore-Louis, K. (2007). Changing the culture of schools: Professional community, organizational learning, and trust. Journal of School Leadership, 16(4), 477-489.

21. Sebastian, J. y Allensworth, E. (2012). The influence of principal leadership on classroom instruction and student learning: A study of mediated pathways to learning. EducationalAdministrationQuarterly, 48(4), 626-663.

22. Sevil-Serrano, J., Abarca-Sos, A., Clemente, J.A.,Murillo-Pardo, B. y García-González, L. (2016). Optimización de variables motivacionales en actividades expresivas en Educación Física. SPORT TK-Revista EuroAmericana de Ciencias del Deporte, 5(2), 103-112.

23. Velázquez-Callado, C. (2015). Aprendizaje cooperativo en Educación Física: estado de la cuestión y propuesta de intervención. RETOS. Nuevas Tendencias en Educación Física, Deporte y Recreación, 28, 234-239.

24. Wood, D. (2011). And Then the basals arrived: School leadership, learning communities and professionalism. International JournalofLeadership in Education, 14(4), 475-497. 